\title{
The Diguanylate Cyclase YfiN of Pseudomonas aeruginosa Regulates Biofilm Maintenance in Response to Peroxide
}

Dept. of Microbiology \& Immunology, Geisel School of Medicine at Dartmouth, Hanover, NH

10 These authors contributed equally to this work.

14 *To whom correspondence should be addressed:

15 Rm 202 Remsen Building

$16 \quad 66$ College Street

17 Geisel School of Medicine at Dartmouth

18 Hanover, NH 03755

19 Phone: (603) 650-1248 (office)

20 email: georgeo@,Dartmouth.edu

22 Running title: YfiN Regulates Biofilm Maintenance

24 Key words: biofilm, c-di-GMP, peroxide, diguanylate cyclase, Pseudomonas aeruginosa 


\section{Abstract}

Pseudomonas aeruginosa forms surface-attached communities that persist in the face of

27 antimicrobial agents and environmental perturbation. Published work has found extracellular

28 polysaccharide (EPS) production, regulation of motility and induction of stress response

29 pathways as contributing to biofilm tolerance during such insults. However, little is known

30 regarding the mechanism(s) whereby biofilm maintenance is regulated when exposed to such

31 environmental challenges. Here, we provide evidence that the diguanylate cyclase YfiN is

32 important for the regulation of biofilm maintenance when exposed to peroxide. We find that,

33 compared to the wild type (WT), static biofilms of the $\Delta y f i N$ mutant exhibit a maintenance

34 defect, which can be further exacerbated by exposure to peroxide $\left(\mathrm{H}_{2} \mathrm{O}_{2}\right)$; this defect can be

35 rescued through genetic complementation. Additionally, we found that the $\Delta y f i N$ mutant

36 biofilms produce less c-di-GMP than WT, and that $\mathrm{H}_{2} \mathrm{O}_{2}$ treatment enhanced motility of surface-

37 associated bacteria and increased cell death for the $\Delta y f i N$ mutant grown as a biofilm compared to

38 WT biofilms. These data provide evidence that YfiN is required for biofilm maintenance by $P$.

39 aeruginosa, via c-di-GMP signaling, to limit motility and protect viability in response to

40 peroxide stress. These findings add to the growing recognition that biofilm maintenance by $P$.

41 aeruginosa is an actively regulated process that is controlled, at least in part, by the wide array of

42 c-di-GMP metabolizing enzymes found in this microbe.

\section{Importance}

We build on previous findings that suggest that $P$. aeruginosa utilizes c-di-GMP

46 metabolizing enzymes to actively maintain a mature biofilm. Here, we explore how the

47 diguanylate cyclase YfiN contributes to the regulation of biofilm maintenance during peroxide 
exposure. We find that mature P. aeruginosa biofilms require YfiN to synthesize c-di-GMP,

49 regulate motility and to insure viability during peroxide stress. These findings provide further

50 evidence that the modulation of c-di-GMP in response to environmental signals is an important

51 mechanism by which biofilms are maintained.

\section{Introduction}

Pseudomonas aeruginosa is a Gram-negative opportunistic pathogen that is found in settings as varied as natural aquatic environments, implanted catheters and the airways of patients afflicted with cystic fibrosis (1-3). P. aeruginosa forms robust biofilms, multicellular surface-attached aggregates that are recalcitrant to antimicrobial therapy and environmental insults $(4,5)$. For motile organisms such as $P$. aeruginosa, biofilms form through a multistep process that requires motility and motility appendages, surface-sensing and the production of extracellular polysaccharides (EPS) (6-9). Flagella mediate initial polar attachment of a cell to a

61 surface, while pili facilitate irreversible surface attachment and microcolony formation $(10,11)$.

62 After surface attachment, the PilY1 protein is secreted to the cell surface where it contributes to surface sensing and the activation of diguanylate cyclase SadC via a Type IV pili (TFP)

64 dependent, outside in signal transduction pathway $(8,12)$. The activation of SadC results in

65 increased bis-(3',5')-cyclic dimeric guanosine monophosphate (c-di-GMP) (12), a second

66 messenger that coordinates surface behavior, virulence and the production of EPS (13-16).

67 Concentrations of c-di-GMP are controlled enzymatically by diguanylate cyclases that synthesize this molecule and phosphodiesterases that degrade this second messenger (17-19). c-di-GMP controls events in biofilm formation beyond surface attachment. For example, once attached to a 
71 with architectural strength for continued growth and protection against predation and environmental challenges, such as antimicrobials, oxidative and osmotic stressors $(4,20-24)$. Biofilms provide protection from a number of insults, including peroxide stress. For example, Acinetobacter oleivorans and Helicobacter pylori $(25,26)$ both respond to peroxides with increased EPS production and enhanced biofilm biomass, while for P. aeruginosa the peroxide $\left(\mathrm{H}_{2} \mathrm{O}_{2}\right)$-responsive OxyR regulates metabolism through the control of iron homeostasis and protein synthesis (27). However, little is known regarding how mature biofilms respond to the exposure to peroxides, including $\mathrm{H}_{2} \mathrm{O}_{2}$, an important issue given that molecules with such chemistry are produced by the immune system $(3,28,29)$.

While early surface attachment events and the processes that drive biofilm maturation

81 (i.e., reversible/irreversible attachment, microcolony formation, EPS production) have received a

82 great deal of attention in the literature, less is known about the regulatory components needed for

83 biofilm maintenance. In previous work in a collaborative effort with the Howell group, we found that RmcA and MorA, phosphodiesterases that break down c-di-GMP, regulate the maintenance of nutrient-limited biofilms (30). To assess if genes that encode diguanylate cyclase activity also contribute to biofilm maintenance, we utilized a mutant library containing clean deletions of all

87 known single-domain DGCs (31). We screened mutants that individually lacked these DGC

88 domains in a static assay to identify deficits in biofilm maintenance. Through this process, we identified YfiN (32) as a putative biofilm maintenance protein. The $y f i N$ gene has previously

90 been found to be a member of the $y f i B N R$ operon (33). Repression of YfiN activity is mediated

91 by its regulator, YfiR. YfiR activity is repressed when bound by YfiB under some conditions

$92(33,34)$. Oxidative stress is thought to play an important role in the regulation of YfiBNR

93 activity, with the crystal structures of YfiR indicating denaturation of this protein in oxidizing 
94 conditions (35) Here, we investigate the role of YfiN in P. aeruginosa motility and biofilm

95 maintenance, and how peroxide stress impacts these biological functions.

96

97

98

99

100

\section{Results}

The $\Delta y f i N$ mutant is defective for biofilm maintenance and susceptible to peroxide stress.

We previously reported the use of a library of in-frame deletion mutants targeting each of $\sim 40$ genes of $P$. aeruginosa PA14 predicted to participate in c-di-GMP synthesis or degradation (31).

We used this library to determine if any of the genes that encode diguanylate cyclases (DGCs) contribute to the ability of $P$. aeruginosa to maintain a biofilm once the community has been established. To test this idea, strains carrying mutations in each of the DGCs of $P$. aeruginosa PA14 were grown statically in 96-well plates for 12h and 48h in M63 biofilm medium (Table S1). As we have described previously, the identification of candidate mutants involved in biofilm maintenance is apparent through a biofilm defect at $48 \mathrm{~h}$, but not at $12 \mathrm{~h} \mathrm{(30).} \mathrm{From} \mathrm{our}$ initial screen of these DGC mutants, we found that that the strain carrying a mutation in the $y f i N$ gene exhibited a biofilm comparable to WT at 12h (Fig. 1A, left panel) but displayed a significant defect at $48 \mathrm{~h}$, with lower amounts of biofilm biomass at this time point (Fig. 1A, middle panel).

To verify that the loss of the biofilm that we observed at the $48 \mathrm{~h}$ assay was dependent on the absence of the $y f i N$ gene, we genetically complemented the $\Delta y f i N$ mutant with a plasmid generously provided by Thomas Wood (32). After induction with arabinose $(0.2 \% \mathrm{~W} / \mathrm{v})$ to induce expression of this gene from the plasmid, we found that biofilms of WT carrying the pMQ70 empty vector $(\mathrm{EV})$, the $\Delta y f i N$ mutant carrying the $\mathrm{EV}$, and $\Delta y f i N$ mutant complemented with pMQ70-yfiN to be comparable at $12 \mathrm{~h}$ (Fig. 1A, left panel). The biofilm defect at $48 \mathrm{~h}$ observed in 
117 the $\Delta y f i N$ mutant carrying the EV can be largely rescued for the $\Delta y f i N$ mutant complemented

118 with pMQ70-yfiN (Fig. 1A, right panel).

\section{Table S1. Diguanylate cyclase mutants screened for defects in biofilm maintenance.}

121 Previous work by $\mathrm{Ha}$ et al. (31) created in-frame, unmarked deletions of all putative diguanylate 122 cyclases that were identified through SMART analyses of proteins encoded in the P. aeruginosa genome (31). Here, these mutants were grown statically in 96-well plates for $12 \mathrm{~h}$ and $48 \mathrm{~h}$ in M63 biofilm medium. Of all mutants screened, only the $\Delta y f i N$ mutant (red) exhibited both comparable biofilm biomass to the WT at $12 \mathrm{~h}$, yet was unable to maintain a biofilm at $48 \mathrm{~h}$. was elicited by starvation or other consequences of prolonged batch culture growth. To test this

129 idea, we repeated the $48 \mathrm{~h}$ static biofilm assay but periodically (every $12 \mathrm{~h}$ ) removed the spent

130 medium, washed the biofilm and added fresh medium. Through this medium replacement assay,

131 we observed that the biofilm defect of the $\Delta y f i N$ mutant was rescued at $48 \mathrm{~h}$ (Fig. 1B, right

132 panel), with the level of biofilm staining comparable to the WT (Fig. 1B, left panel). 
A

$12 \mathrm{~h}$

$48 \mathrm{~h}$

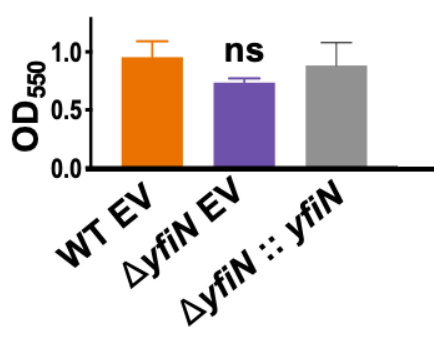

B $\quad 12 h$

$12 \mathrm{~h} \quad 48 \mathrm{~h}$
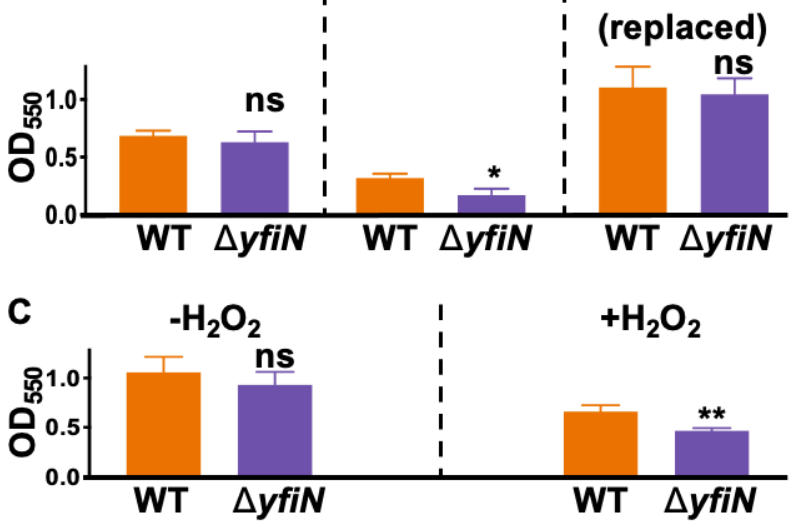

Figure 1. The $\Delta y$ fiN mutant is defective for biofilm maintenance and a pre-formed biofilm is reduced when exposed to peroxide. A) Biofilm formation by the WT, the WT carrying the pMQ70 empty vector (EV), the $\Delta y f i N$ mutant carrying the $\mathrm{EV}$, and $\Delta y f i N$ mutant complemented with pMQ70-yfiN after $12 \mathrm{~h}$ and $48 \mathrm{~h}$ (left and right panel, respectively). The biofilm formed was assessed by staining with crystal violet $(\mathrm{CV})$, the $\mathrm{CV}$-stained biofilm solubilized in $30 \%$ glacial acetic acid and the absorbance (OD 550 ) measured. B) Biofilm formation by WT and the $\Delta y f i N$ mutant was assessed in a 96-well plate assay. The strains were grown on M63 medium supplemented with arginine $(0.4 \% \mathrm{w} / \mathrm{v})$ and the biofilm measured after $12 \mathrm{~h}$ (left panel), $48 \mathrm{~h}$ (middle panel), or after $48 \mathrm{~h}$ wherein the medium was replaced every $12 \mathrm{~h}$ (right panel). The quantification of the biofilm by $\mathrm{CV}$ was performed as described in panel A. C) Biofilms of WT and the $\Delta y f i N$ mutant were grown for $12 \mathrm{~h}$, after which medium was replaced with control growth medium (left panel) or medium supplemented with $50 \mathrm{mM} \mathrm{H}_{2} \mathrm{O}_{2}\left(+\mathrm{H}_{2} \mathrm{O}_{2}\right)$ for an additional 12h of growth. Biofilm formation was assessed as described in panel A. For all panels, error bars represent standard deviation of the results from three biological replicates each performed with three technical replicates. Statistical significance was assessed via Student's T-test in all panels except 1A, in which Dunnett's multiple comparisons post-test was used. *, **, indicate differences that are significantly different at $\mathrm{P}<0.05$ and $\mathrm{P}<0.01$, respectively, compared to the

150 WT. ns; non-significant. 
154 hypothesized that the biofilm maintenance defect manifested by the $\Delta y f i N$ mutant may be due to

155 an altered response to peroxide stress. To test this hypothesis, we compared the amount of

156 biofilm retained by WT or the $\Delta y f i N$ mutant after $24 \mathrm{~h}$ with a single medium replacement at $12 \mathrm{~h}$,

157 to biofilms of these same strains exposed to $50 \mathrm{mM} \mathrm{H}_{2} \mathrm{O}_{2}$ added to the replacement medium.

158 After 24h with control replacement medium, biofilms of WT and the $\Delta y f i N$ mutant were not

159 significantly different (Fig. 1C, left panel). However, when replacement medium contained 50

$160 \mathrm{mM} \mathrm{H}_{2} \mathrm{O}_{2}$ we observed that biofilms of the $\Delta y f i N$ mutant exhibited a significantly lower biofilm

161 biomass compared to the WT (Fig. 1C, right panel). Collectively, these results suggest that YfiN

162 may be important for biofilm maintenance and that $P$. aeruginosa biofilms lacking this DGC

163 exhibited reduced biofilm biomass when exposed to peroxide.

Dispersion and cell death contribute to the $\Delta y f i N$ mutant biofilm maintenance defect. The

167 above results provide evidence that YfiN impacts biofilm maintenance in the face of peroxide

168 stress. In order to determine biomass and cell viability before and after treatment, static biofilms

169 were grown in glass-well dishes containing the same medium used in the 96-well dish assay to

170 directly image the biofilms. The biofilm biomass was determined by measuring the fluorescence

171 of the WT and the $\Delta y f i N$ mutant strain carrying the multicopy plasmid pSMC21, which

172 constitutively expresses GFP (ex500/em513). To visualize dead cells, the biofilms were washed

173 with the same culture medium containing propidium iodide (PI) immediately prior to imaging.

174 Fluorescence intensity was used to quantify the biofilm biomass of each strain (green

175 fluorescence), and the ratio of PI to GFP intensity was used as a metric to measure normalized 176 cell death within the biofilms. 
189 in part, to increased cell death in this mutant.
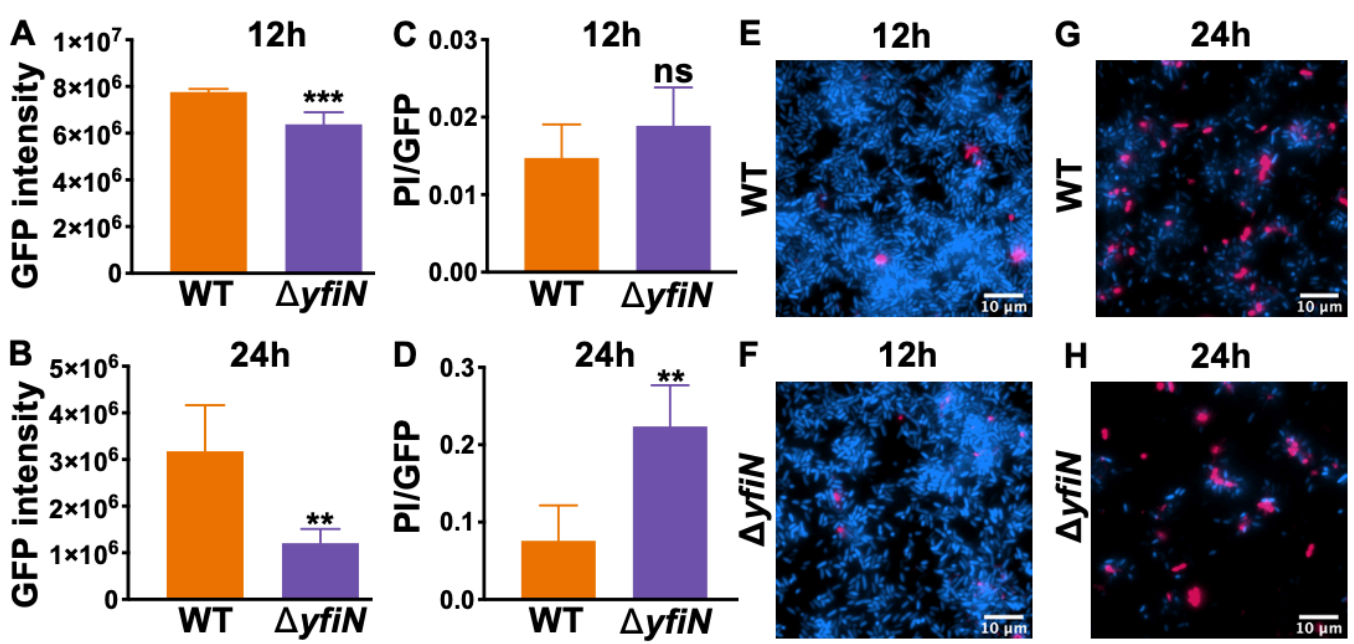

Figure 2. Cell death likely contributes to the $\Delta y f i N$ mutant biofilm maintenance defect.

191 Biofilm biomass and cell death was assessed microscopically in glass-bottom dishes with WT and the $\Delta y f i N$ mutant. Cell biomass was determined using fluorescence mediated by the plasmid pSMC21, which encodes a constitutively-expressed GFP. Cell viability was determined as a ratio

194 of propidium iodide (PI)-stained (dead) cells to GFP-mediated green fluorescence (live cells).

195 The PI was added to the dishes immediately prior to visualizing, and the cells were washed to

196 remove excess dye. A-B) WT and the $\Delta y$ fiN mutant biofilm biomass was quantified by 
measuring GFP intensity at $12 \mathrm{~h}(\mathrm{~A})$ and $24 \mathrm{~h}(\mathrm{~B})$. We observed that the biofilm maintenance defect of the $\Delta y f i N$ mutant occurred as early as $24 \mathrm{~h}$ in the glass-bottom plates assay and consequently used this point for the analysis here. C-D) Cell death was measured as a ratio of PI staining to GFP intensity at $12 \mathrm{~h}$ (C) and $24 \mathrm{~h}$ (D). For all panels, error bars represent standard deviations of the results from three biological replicates each performed with 6 technical replicates. Statistical significance was assessed via a Student's T-test. **,***, indicate differences that are significantly different at $\mathrm{P}<0.01$ and $\mathrm{P}<0.001$ respectively, compared to the WT. ns, non-significant. (E-H) Representative images showing combined GFP (blue) and PI staining (pink) of WT biofilm at $12 \mathrm{~h}(\mathrm{E})$ and $24 \mathrm{~h}(\mathrm{G})$, and the $\Delta y$ fiN mutant biofilm at $12 \mathrm{~h}(\mathrm{~F})$

206 and $24 \mathrm{~h}(\mathrm{H})$.

\section{The $\Delta y f i N$ mutant biofilm exhibits increased susceptibility to peroxide stress under flow.}

209 The static biofilm data presented thus far are consistent with the hypothesis that YfiN contributes

to biofilm maintenance and that the loss of this DGC enhances cell death as these communities

211 age and/or encounter oxidative stress in the form of peroxide. We next wanted to determine the

212 impact of $\mathrm{H}_{2} \mathrm{O}_{2}$ specifically on the $\Delta y f i N$ mutant biofilm under flow conditions, which helps

213 mitigate the presence of potential confounding factors, such as accumulating metabolites in the

214 extracellular milieu. To address this question, we pursued a microfluidics-based approach in

215 which WT and the $\Delta y f i N$ mutant with constitutive expression of GFP (using pSMC21, described

216 above) were grown as a biofilm under flow in biofilm medium, a buffered minimal medium

217 containing $1.0 \mathrm{mM} \mathrm{K}_{2} \mathrm{HPO}_{4}, 0.6 \mathrm{mM} \mathrm{MgSO}_{4}$ and $0.4 \%$ arginine, $\mathrm{pH}=7.5$. This medium is

218 similar to the M63-based medium used in the static assay but has been optimized for use in flow

219 studies.

First, to determine if the loss of YfiN adversely impacted the ability of this mutant to

221 form a biofilm under standard flow conditions (i.e., no $\mathrm{H}_{2} \mathrm{O}_{2}$ added), we compared GFP intensity

222 with that of WT over a $12 \mathrm{~h}$ time course kinetic assay. The WT and $\Delta y f i N$ mutant biofilms were

223 comparable for the first 10h of this assay, after which WT biofilm was modestly but significantly

224 higher than that of $\Delta y f i N$ mutant in three of the last five time points assessed after 10h (Fig. S1). 


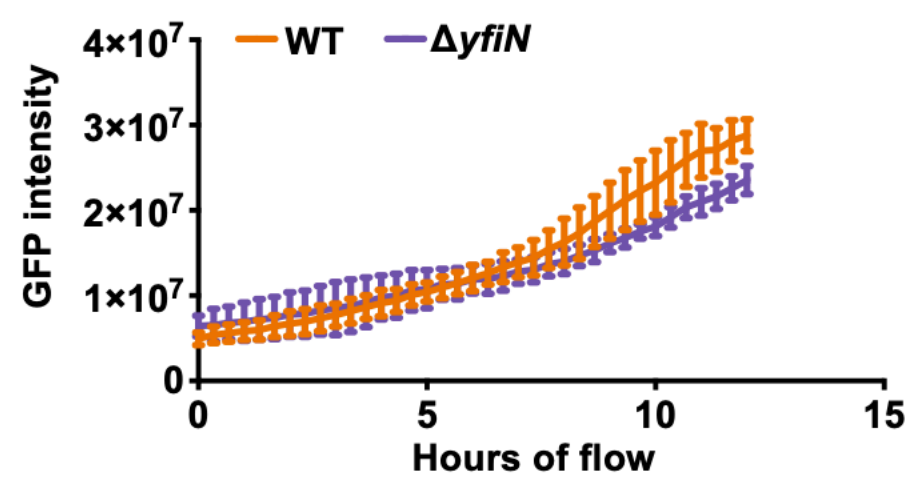

226 Figure S1. Initial biofilm formation by WT and the $\Delta y \boldsymbol{f i N}$ mutant under flow. The WT strain

227 and the $\triangle y f i N$ mutant carrying pSMC21, a plasmid which constitutively expresses GFP, were

228 inoculated into microfluidic chambers and allowed $1 \mathrm{~h}$ to attach prior to beginning flow of

229 biofilm medium. Flow was resumed, then GFP fluorescence was measured every 20 minutes for

230 12h. Error bars represent standard deviations of the results from three biological replicates each

231 performed with three technical replicates. Statistical significance was assessed via Sidak's

232 multiple comparisons post-test. The WT formed significantly more biofilm at 10.7, 11 and $11.7 \mathrm{~h}$.

A
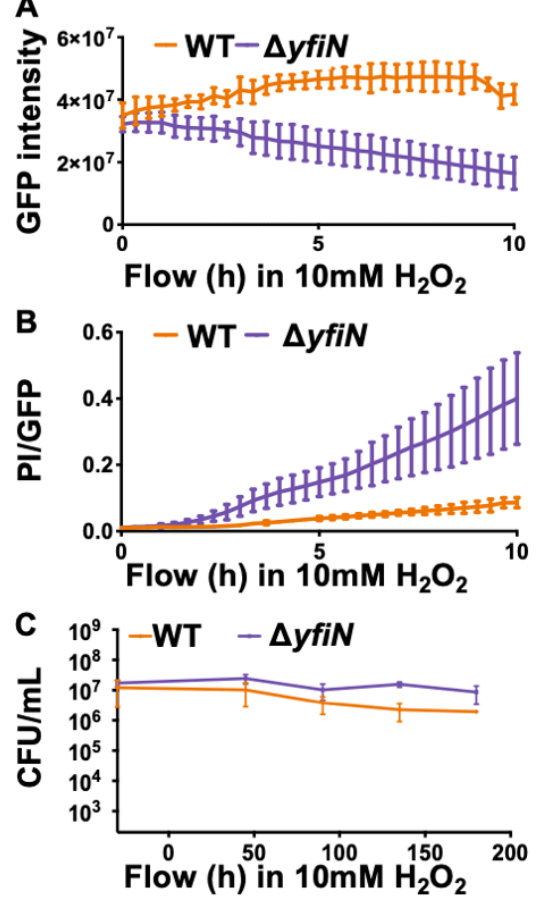

D

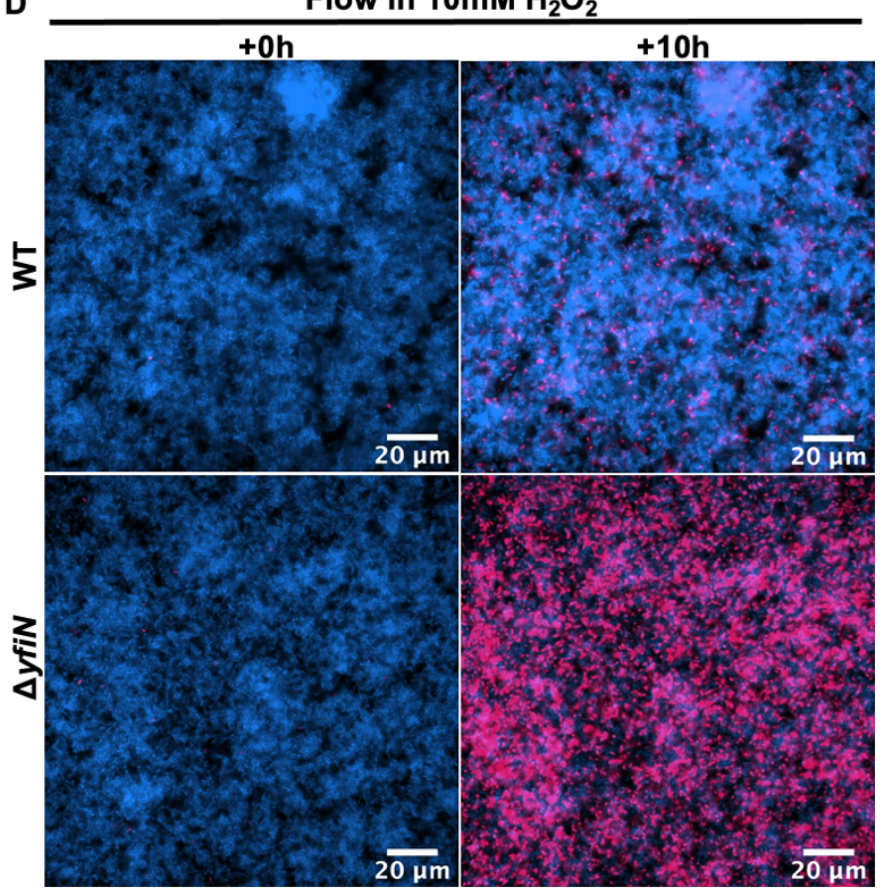

233 Figure 3. The $\Delta y f i N$ mutant biofilm exhibits increased cell death and dispersal in response

234 to peroxide treatment. A) Biofilm formation by the WT and the $\Delta y f i N$ mutant was quantified

235 through fluorescent microscopy and GFP signal intensity measured for $10 \mathrm{~h}$ after the addition of

$236 \mathrm{H}_{2} \mathrm{O}_{2}$. B) Cell death was measured as a ratio of PI to GFP over the same time course as panel A.

237 C) WT and the $\triangle y f i N$ mutant carrying plasmid pSMC21 encoding GFP were inoculated into a

238 microfluidics device and grown in biofilm medium. After 14h of growth, medium was replaced

239 with biofilm medium containing $10 \mathrm{mM} \mathrm{H}_{2} \mathrm{O}_{2}$ and PI. Bacterial CFUs/mL were measured in the 
240 effluent $30 \mathrm{~min}$ prior to and up to $180 \mathrm{~min}$ after being exposed to $\mathrm{H}_{2} \mathrm{O}_{2}$. Error bars represent 241 standard deviations of the results from three biological replicates each performed with three 242 technical replicates. Statistical significance was assessed via Sidak's multiple comparisons post-

243 test. Compared to WT, results from the $\Delta y f i N$ mutant were significantly different $(\mathrm{P}<0.05)$ from 244 WT after 6h in Figure 3A, 3h in Figure 3B, and at $45 \mathrm{~min}$ and $120 \mathrm{~min}$ in Figure 3C. D)

245 Representative images from microfluidics studies used to generate the quantitative data 246 presented in panels A and B. Viable cells (GFP) are colored blue while PI-stained cells are 247 colored magenta.

We first assessed whether the $\Delta y f i N$ mutant was more susceptible to starvation than the

250 WT, as was reported for strains carrying mutations in the $r m c A$ or mor $A$ genes (30); we observed

251 no such phenotype in the microfluidic devices (not shown). To determine whether the $\Delta y f i N$

252 mutant was more susceptible to $\mathrm{H}_{2} \mathrm{O}_{2}$ under flow compared to the WT, both strains were grown

253 for $14 \mathrm{~h}$ as a biofilm, after which influent biofilm medium was replaced with medium containing

$25410 \mathrm{mM} \mathrm{H}_{2} \mathrm{O}_{2}$, as well as PI to stain dead cells, and the biofilms imaged over an additional $12 \mathrm{~h}$

255 time course. We then assessed biofilm biomass, cell viability and cell dispersion after this $12 \mathrm{~h}$

256 exposure to peroxide.

257 Using GFP signal intensity as a measure of biofilm biomass, we found that prior to the

258 addition of $\mathrm{H}_{2} \mathrm{O}_{2}$, there was no significant difference in the biofilm biomass of the WT versus the

$259 \Delta y f i N$ mutant over the first 10 h of the experiment (Fig. S1), indicating that both strains could

260 form a robust biofilm. We did note a difference in the biofilm biomass between these strains

261 under flow after $12 \mathrm{~h}$, but this reduction was modest and not significant at every time point (see

262 Fig. S1). Monitoring the biofilm biomass showed that the addition of $\mathrm{H}_{2} \mathrm{O}_{2}$ resulted in a

263 significant decrease in $\Delta y f i N$ biofilm biomass $6 \mathrm{~h}$ after treatment compared to the WT, which

264 continued to increase in biomass as judged by GFP signal intensity (Fig. 3A, D). Our analysis

265 shows that $\mathrm{H}_{2} \mathrm{O}_{2}$ treatment adversely impacted the viability of $\Delta y f i N$ mutant; this mutant 
exhibited a significant increase in its PI/GFP ratio compared to WT starting $3 \mathrm{~h}$ after $\mathrm{H}_{2} \mathrm{O}_{2}$

treatment and at each subsequent time point (Fig. 3B, D).

To determine if $\mathrm{H}_{2} \mathrm{O}_{2}$ exposure caused cells to disperse from the biofilm, colony forming units (CFUs) were measured in the effluent 30 min prior to and for $3 \mathrm{~h}$ after the $\mathrm{H}_{2} \mathrm{O}_{2}$-containing medium switch. Prior to exposure to $\mathrm{H}_{2} \mathrm{O}_{2}$, cell dispersal between WT and mutant $\Delta y f i N$ were

271 not significantly different. However, the switch to medium containing $10 \mathrm{mM} \mathrm{H}_{2} \mathrm{O}_{2}$ resulted in

272 modest increases of $\Delta y f i N$ mutant cells in the effluent, and this difference was significant at 45

273 and 120 min post-stress exposure (Fig. 3C). Collectively, the use of static and microfluidics assays showed that the loss of YfiN function resulted in increased cell death, as well as a modest

275 increase in dispersal of the biofilm cells compared to the WT.

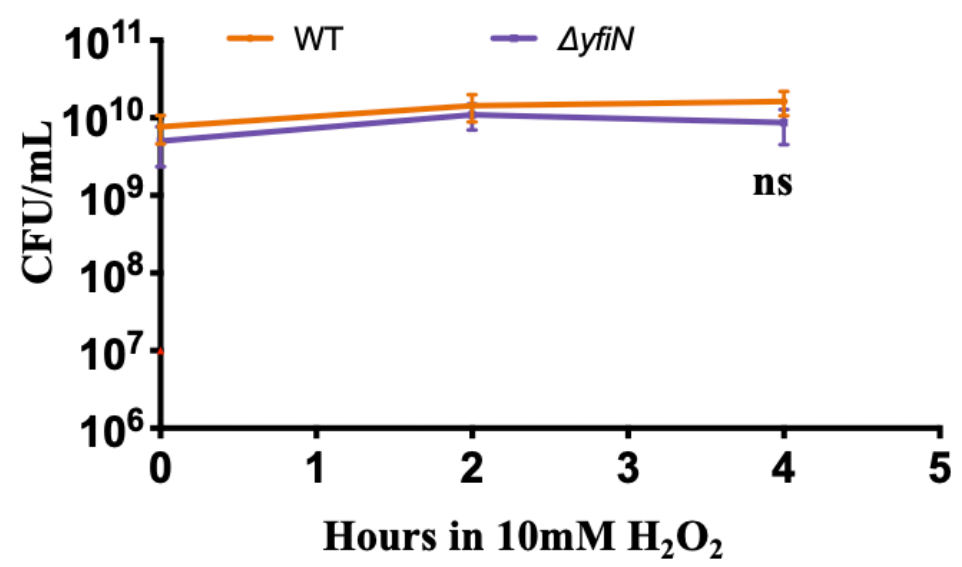


284 a normalized $\mathrm{OD}_{600}$ of 0.5 . CFU/mL were measured prior to and 2-4h after the addition of $\mathrm{H}_{2} \mathrm{O}_{2}$.

285 Error bars represent standard deviation of the results from three biological replicates each

286 performed with three technical replicates. Statistical significance was assessed with a Sidak's

287 multiple comparisons post-test; ns, indicates a non-significant.

\section{Loss of YfiN impacts c-di-GMP levels and motility in response to peroxide stress. Y fiN}

290 contains a DGC domain that has been shown to mediate the synthesis of c-di-GMP $(32,33)$. We

291 next assessed whether exposure to $\mathrm{H}_{2} \mathrm{O}_{2}$ impacted c-di-GMP levels. To measure c-di-GMP level,

292 we used a $\mathrm{P}_{c d r A}-g f p$ promoter fusion on a plasmid to assess the transcriptional activity of $c d r A$, a

293 gene that is positively regulated by c-di-GMP. We normalized the GFP signal to biomass using

$294 \mathrm{mKO}(\mathrm{ex} 548 / \mathrm{em} 559)$ inserted as a double copy at the att site on the chromosome and expressed

295 constitutively. Bacteria were inoculated into glass-bottom dishes, grown as a monolayer of

296 surface-attached cells, exposed to $1 \mathrm{mM} \mathrm{H}_{2} \mathrm{O}_{2}$ (a concentration of peroxide that is well below the

297 toxic levels analyzed above) and then imaged over the next $8 \mathrm{~h}$. Consistent with previous

298 findings, the loss of Y fiN reduced c-di-GMP levels, evident in the lower GFP signal (pink in

299 images) for the $\Delta y f i N$ mutant compared to WT (Fig 4A, top left and bottom left, respectively,

300 and Fig. 4B, time = 0). After exposure to peroxide, we observed an initial drop in GFP intensity

301 by both WT and the $\Delta y f i N$ mutant (Fig. 4B), indicative of less c-di-GMP in response to hydrogen

302 peroxide. Interestingly, while WT exhibited a recovery in c-di-GMP after $\sim 6 \mathrm{~h}$, this recovery was

303 not observed in the $\Delta y f i N$ mutant, for which the GFP signal remained low. 

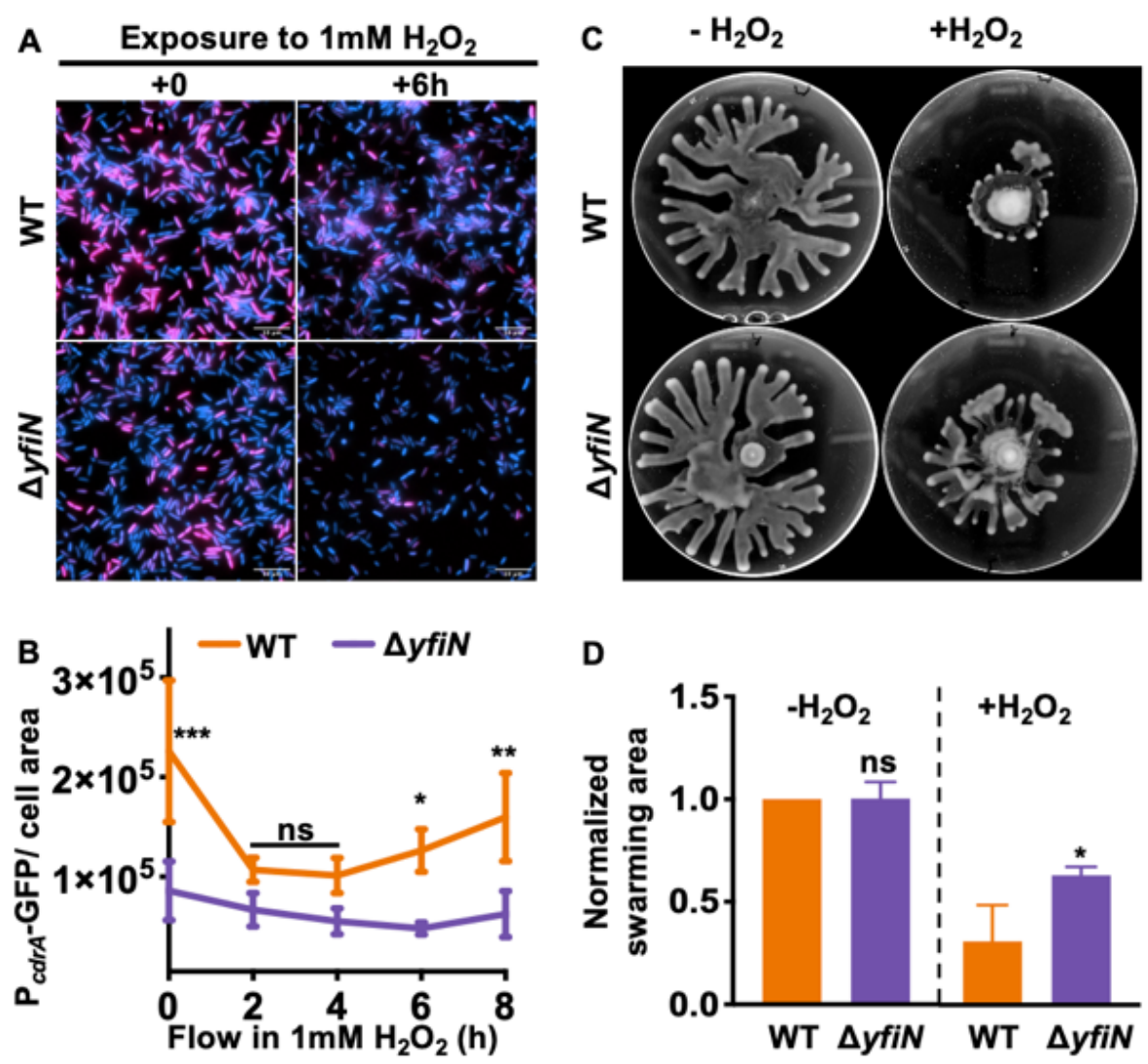

Figure 4. Loss of YfiN impacts c-di-GMP levels and motility in response to peroxide stress. A) The WT strain and the $\Delta y f i N$ mutant carrying the $\mathrm{P}_{c d r A}-g f p$ fusion expressed from a multicopy plasmid were grown in glass bottom 8-well dishes containing M63 medium for $3 \mathrm{~h}$ prior to the addition $1 \mathrm{mM} \mathrm{H}_{2} \mathrm{O}_{2}$, then imaged over $8 \mathrm{~h}$ with a representative image from the $0 \mathrm{~h}$ and $6 \mathrm{~h}$ time points shown. The pink color shows GFP expression, and the blue color is contributed by the constitutively expressed mKO fluorescent protein. (B) Quantification of GFP signal intensity for the strains described in panel A. Fluorescent microscopy was used to determine GFP signal intensity as a measure of c-di-GMP production and normalized to the surface area of cells that constitutively expressed $\mathrm{mKO}$ fluorescent protein. Statistical significance was assessed with a Sidak's multiple comparisons post-test. C) Representative images of the indicated strains grown on swarm assay plates $16 \mathrm{~h}$ after inoculation, with and without $5 \mathrm{mM} \mathrm{H}_{2} \mathrm{O}_{2}$. D) Quantification of plates described in panel $\mathrm{C}$; all values are plotted after normalization to the WT not treated with $\mathrm{H}_{2} \mathrm{O}_{2}$, which was set to a value of 1 . For panels $\mathrm{B}$ and $\mathrm{D}$, error bars represent standard deviations of the results from three biological replicates each performed with three technical replicates. *, $* *, * * *$ indicate differences that are significantly different at $\mathrm{P}<0.05, \mathrm{P}<0.01$, and $\mathrm{P}<0.0001$ respectively, compared to the WT. ns, non-significant. 
Two key mechanisms whereby c-di-GMP regulates biofilm formation and maintenance

Movies S1 and S2). Thirty minutes after the addition of $\mathrm{H}_{2} \mathrm{O}_{2}$, the motility of the $\Delta y f i N$ mutant

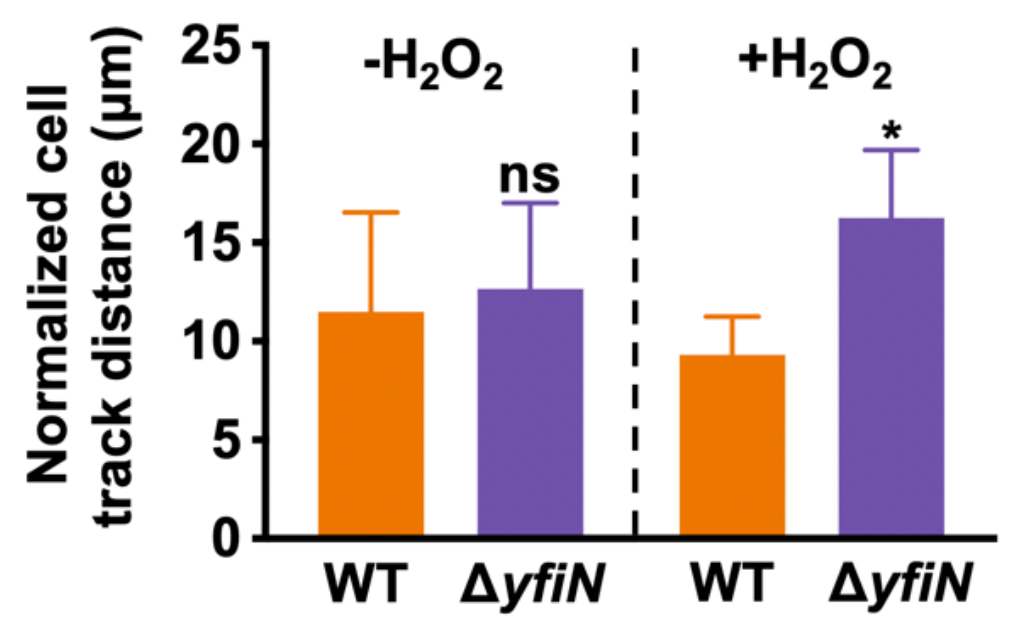
WT strain and the $\Delta y f i N$ mutant were inoculated into glass well dishes for $1 \mathrm{~h}$. Motility was assessed over a 5 minute time course using brightfield microscopy prior to and 30 minutes after the addition of $5 \mathrm{mM} \mathrm{H}_{2} \mathrm{O}_{2}$. The TrackMate program was used to measure the tracks that each bacterium traveled over time, and the sum distance of all cell tracks was normalized to the number of bacteria at the start of imaging. Statistical significance was assessed via a Student's T-test. * indicates differences that are significantly different at $\mathrm{P}<0.05$, compared to the WT. ns, non-significant. 


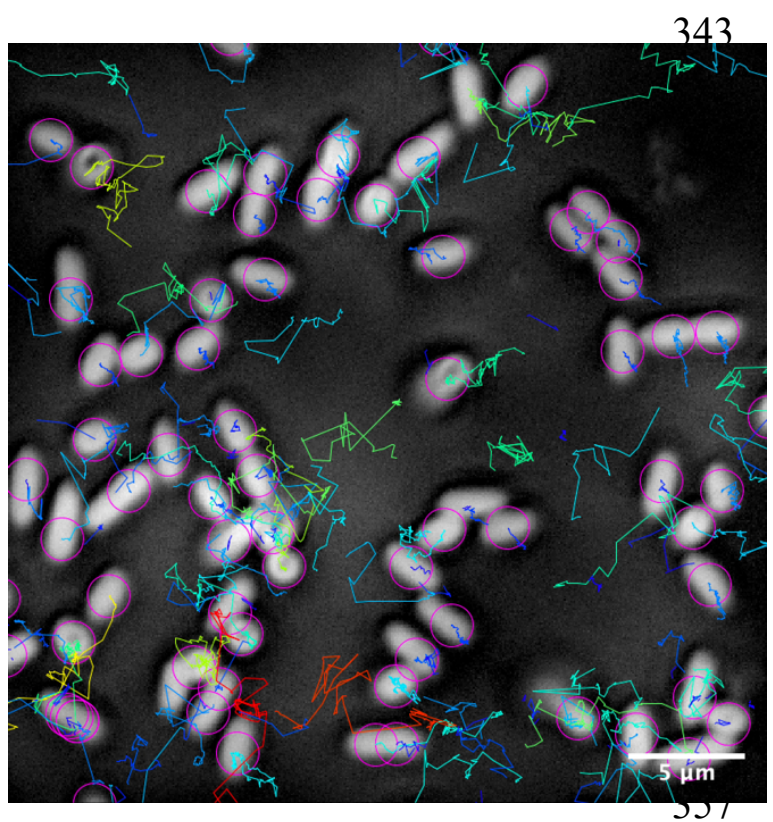

358 Movie S1. Motility of WT prior to the addition of $\mathbf{H}_{2} \mathbf{O}_{2}$. WT was inoculated into a glass well

359 dish. Surface-attached bacteria were imaged one hour after inoculation with brightfield

360 microscopy over a 5 minute time-course. The TrackMate program was used to identify bacteria

361 (purple circles) and the distance of the tracks that bacteria traveled over time was measured and 362 bacterial tracked distance depicted as a color-coded spectrum (blue $<10 \mu \mathrm{m}$; red $>30 \mu \mathrm{m}$ ).

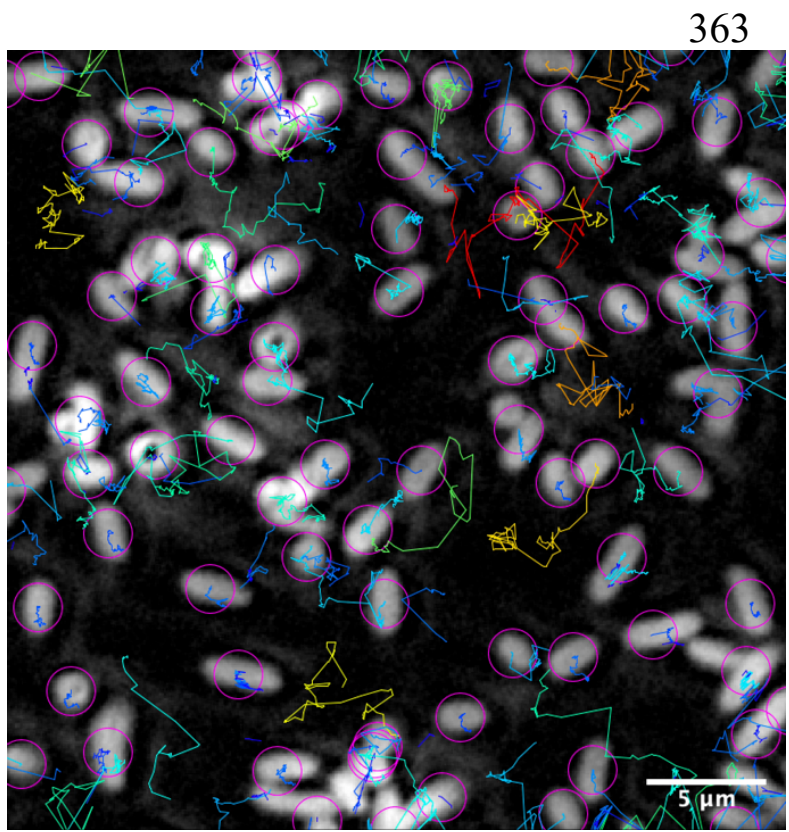

378 Movie S2. Motility of the $\Delta y \boldsymbol{y}$ iN mutant prior to the addition of $\mathbf{H}_{\mathbf{2}} \mathbf{O}_{2}$. The $\Delta y f i N$ mutant was 379 inoculated into a glass well dish and surface-attached bacteria were imaged $1 \mathrm{~h}$ after inoculation, 380 as described in Movie S1. The distance of the tracks that bacteria traveled over time was 381 measured and bacterial tracked distance depicted as a color-coded spectrum (blue $<10 \mu \mathrm{m}$; $382 \mathrm{red}>30 \mu \mathrm{m})$. 


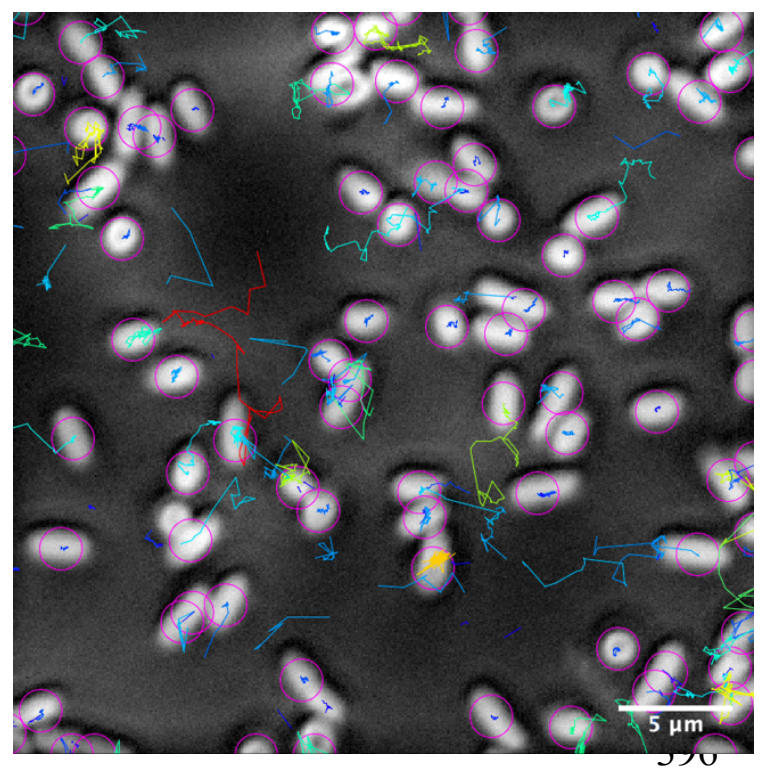

Movie S3. Motility of WT after the addition of $\mathrm{H}_{2} \mathbf{O}_{2}$. WT was inoculated into a glass well dish 398 and allowed to attach to the surface for $1 \mathrm{~h}$. Bacteria were subsequently exposed to $5 \mathrm{mM} \mathrm{H}_{2} \mathrm{O}_{2}$ 399 and imaged after 30 minutes for a 5 minute time-course using brightfield microscopy. The 400 distance of the tracks that bacteria traveled over time was measured and bacterial tracked 401 distance depicted as a color-coded spectrum (blue $<10 \mu \mathrm{m}$; red $>30 \mu \mathrm{m}$ ).

402

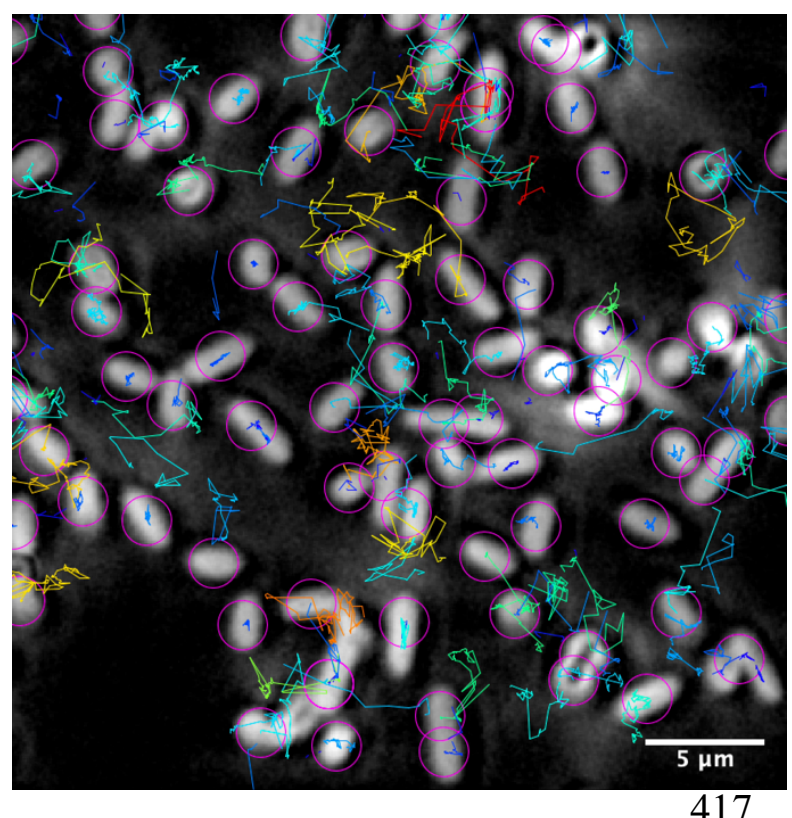

418 Movie S4. Motility of the $\Delta y f i N$ mutant after the addition of $\mathrm{H}_{2} \mathrm{O}_{2}$. The $\Delta y f i N$ mutant was 419 inoculated into a glass well dish and surface-attached bacteria were imaged $1 \mathrm{~h}$ after inoculation 420 as described in Movie S1. The distance of the tracks that bacteria traveled over time was 421 measured and bacterial tracked distance depicted as a color-coded spectrum (blue $<10 \mu \mathrm{m}$; $422 \operatorname{red}>30 \mu \mathrm{m})$. 
We next assayed swarming on $0.5 \%$ agar plates in response to $+/-5 \mathrm{mM} \mathrm{H}_{2} \mathrm{O}_{2}$. Swarming

motility by $P$. aeruginosa is a surface-based motility which utilizes the flagellum. In the absence

426 of $\mathrm{H}_{2} \mathrm{O}_{2}$, both strains exhibit comparable swarm motility (Fig 4C-D, left panels). While the

427 addition of $5 \mathrm{mM} \mathrm{H}_{2} \mathrm{O}_{2}$ resulted in almost complete suppression of swarming motility by the WT,

428 the $\Delta y f i N$ mutant exposed to $\mathrm{H}_{2} \mathrm{O}_{2}$ still displayed swarming motility, although somewhat reduced

429 from the no treatment control (Fig 4C-D, compare right panels to left panels). Collectively, these

430 results suggest that $P$. aeruginosa uses YfiN to modulate c-di-GMP levels and suppress motility

431 when exposed to peroxide stress.

433 Likely YfiBNR homologs are found in other bacterial genera. Known YfiN homologs are

434 present in other biofilm-forming, Gram-negative bacteria like Pseudomonas fluorescens,

435 Escherichia coli, and Yersinia pestis (36-39). Given the above evidence that YfiN regulates

436 biofilm maintenance in P. aeruginosa, we mined GenBank for YfiBNR homologs in other

437 bacteria, with a particular focus on Gram-negative, biofilm forming microbes. To identify

438 potential homologs, we used the nucleotide sequence of the $P$. aeruginosa YfiBNR operon as a

439 query in an NCBI Nucleotide BLAST search against the RefSeq representative genomes

440 database (40). We aligned the 280 sequences with $>75 \%$ query cover via Multiple Alignment

441 using Fast Fourier Transform (MAFFT), then mapped sequences into a phylogenetic tree using

$442 \quad$ FastTree 2.1.11 (41, 42).

The resulting phylogenetic tree (summarized in Fig. 5A) does not group by taxonomic

444 classification and contains 116 species of bacteria in eight genera: $83.2 \%, 7.9 \%$, and $3.9 \%$ of the

445 hits were Pseudomonas, Janithobacterium, and Serratia, respectively. Cupriavidus, Rugamonas, 
Inquilinus, Bordetella, and Achromobacter each made up $0.4-1.4 \%$ of the remaining sequences.

447 The resulting phylogenetic tree identified a cluster (Fig. 5B) with high bootstrap support that

448 formed around the query sequence. This cluster contains Pseudomonas and Serratia, which are

449 genera of different orders. Notably, the cluster includes a sequence from Serratia marcescens, a

450 known biofilm-forming, opportunistic human pathogen that infects the respiratory tract, urinary

451 tract and eyes (43-45). These YfiBNR homologs may be similarly involved in biofilm

452 maintenance and response to peroxide stress in their respective species.

A

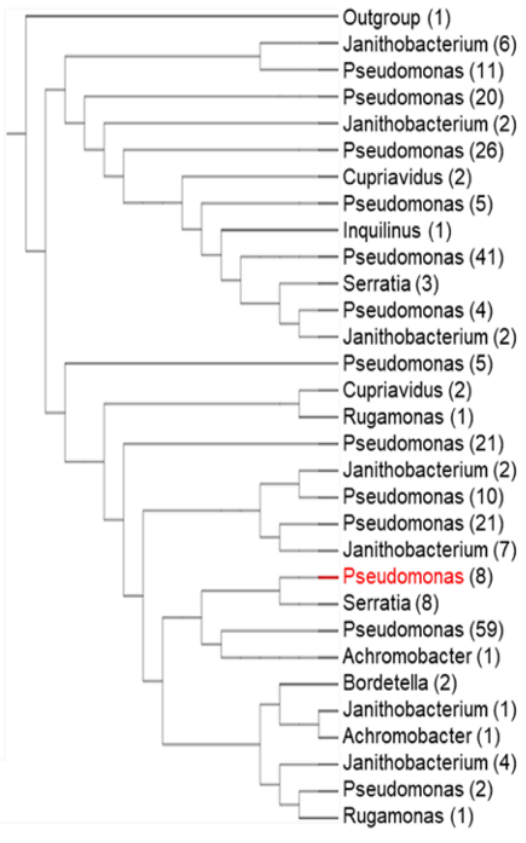

B

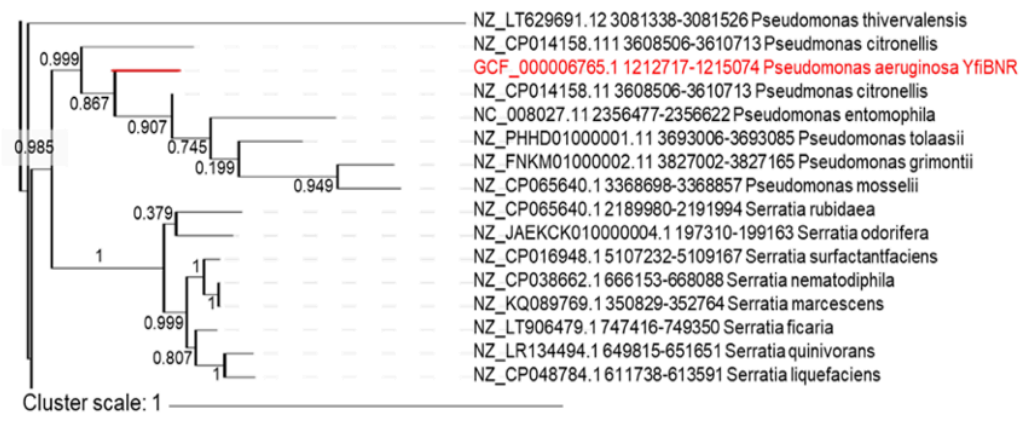

Figure 5: YfiBNR homologs are found in multiple bacterial genera. A) Potential YfiBNR homologs were identified via a Nucleotide BLAST search, aligned with MAFFT, arranged into a phylogenetic tree using FastTree 2.1.11, and visualized using Interactive Tree of Life software (46). Sequences most closely mapped to sequences of the same genus were collapsed to generate the above abbreviated tree. The full tree is shown in Figure S4. Each branch is labeled with the number of sequences from that genus present on that branch in the full tree. The branch containing the $P$. aeruginosa YfiBNR query sequence is marked in red. Branch lengths are not mapped on this tree. B) A cluster labeled with high bootstrap values, indicating frequent mapping of the same branches in various iterations of the tree, formed around the YfiBNR query sequence, which is marked in red. Branch lengths, which mark how much genetic divergence exists between sequences, are to scale. The sequences mapped most closely to YfiBNR were 
bioRxiv preprint doi: https://doi.org/10.1101/2021.07.29.454409; this version posted July 30, 2021. The copyright holder for this preprint (which

was not certified by peer review) is the author/funder, who has granted bioRxiv a license to display the preprint in perpetuity. It is made available under aCC-BY-NC 4.0 International license.

465 from multiple other Pseudomonas and Serratia strains, suggesting potential homology across 466 species and genera.

467

468

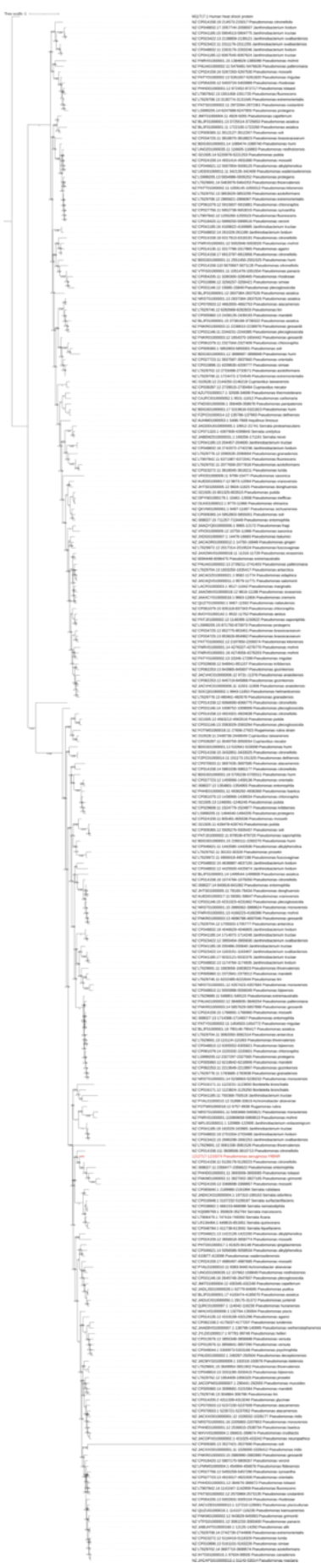

469 Figure S4. Full phylogenetic tree of potential YfiBNR homologs. The full phylogenetic tree of 470 potential YfiBNR homologs was produced from 280 aligned sequences from 116 species in eight 471 genera. 


\section{Discussion}

YfiN homologs are found among various bacterial genera, including Pseudomonas,

474 Escherichia, Salmonella and Serratia, and this protein has been shown to play a role in

475 regulating intracellular c-di-GMP in $P$. aeruginosa here and in previous studies $(32,47)$. The

476 contributions of YfiN have been largely studied in the context of motility and biofilm formation,

477 whereby a loss of YfiN leads to less c-di-GMP and reduced biofilm formation $(32,48)$,

478 consistent with our results in $P$. aeruginosa. Increased motility and the resulting dispersal of the

479 biofilm that we observe here has been indirectly supported through the study of upstream

480 regulators. For instance, deletion of $\mathrm{YfiR}$, an upstream inhibitor of $\mathrm{Y}$ fiN, has been shown to

481 repress motility in $P$. aeruginosa PAO1 (33). A release of inhibition by YfiR leads to activation

482 of the c-di-GMP-producer YfiN, thus reducing motility. Furthermore, previous biochemical

483 studies have shown that oxidized glutathione disrupts disulfide bond formation and protein

484 stability of YfiR (35), suggesting the critical role of redox stress on the regulation of this

485 pathway.

Here we observe a significant biofilm maintenance defect at $48 \mathrm{~h}$, but not $12 \mathrm{~h}$, in the 
aeruginosa, with our group and many others contributing to the known mechanisms $(12,18,49$,

496 50). We presume that c-di-GMP-mediated regulation of motility is through one of the known

497 mechanisms, but this remains to be demonstrated. Why we observe increased cell death in the

$498 y f i N$ mutant treated with peroxide is a mystery. Our previous work strongly suggested that loss

499 of RmcA/MorA contributed to cell death during starvation because strains lacking these proteins

500 continued to use resources to make EPS even when starving; the mutants were likely starving

501 themselves to death (30). Here, we suggest two possibilities for the cell death observation. The

502 first is that high c-di-GMP levels promoted by YfiN are necessary to induce protective responses

503 to peroxide. Increased c-di-GMP in Vibrio cholerae has been found to induce matrix production

504 and enhance catalase activity $(51,52)$. In further support of this view in $P$. aeruginosa, the DGC

505 PA3177 has been found to synthesize c-di-GMP in response to oxidative hypochlorite (53) and

506 to mediate $\mathrm{H}_{2} \mathrm{O}_{2}$ and antibiotic tolerance (54). Furthermore, sublethal concentrations of $\mathrm{H}_{2} \mathrm{O}_{2}$

507 have been shown to select for mutations in the gene encoding for WspF, which represses the

508 DGC WspR (55). The second possibility is that exposure to peroxide in the absence of YfiN

509 results in the biofilm bacteria returning to a less tolerant, planktonic lifestyle. This second

510 hypothesis is supported by the observation that YfiN mutants are more motile and more readily

511 dispersed from the biofilm subsequent to peroxide exposure. 


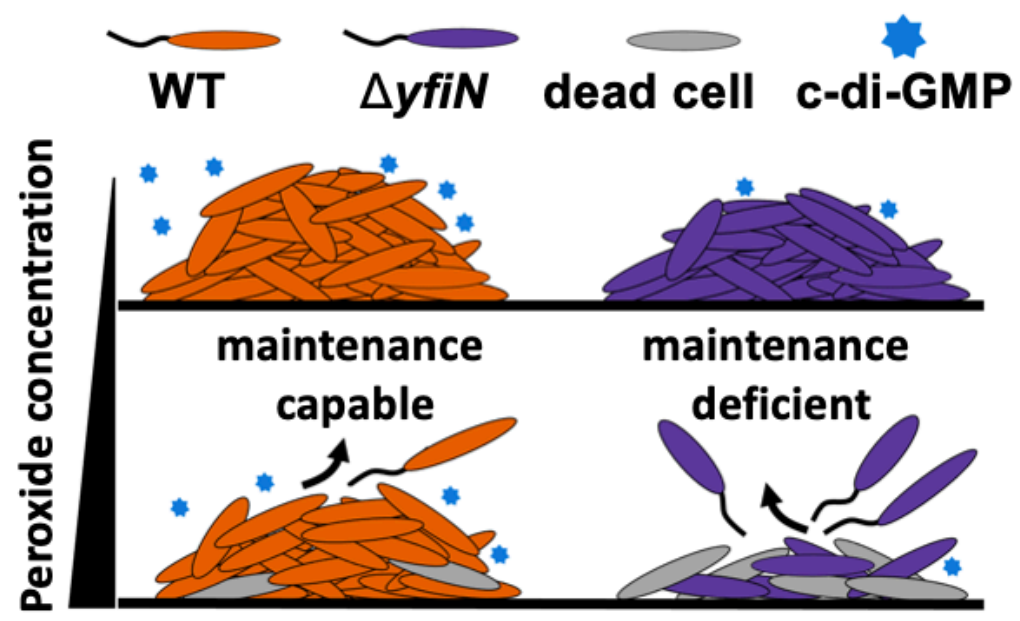

513 Figure 6: Model for P. aeruginosa Y fiN's contribution to biofilm maintenance in response to

514 peroxide exposure. YfiN is not a major contributor to early biofilm formation in our growth

515 conditions (top panel). In a mature WT biofilm (bottom panel), YfiN is needed to regulate

516 maintenance through the modulation of c-di-GMP and motility in response to peroxide. The loss

517 of Y fiN results in biofilms that are maintenance-deficient and exhibit increased cell death and

518 dispersal upon exposure to exogenous peroxide, and perhaps in response to endogenous redox

519 active molecules.

520

Interestingly, we found that loss of biofilm biomass in later-stage biofilms in strains

522 lacking YfiN function in both the absence and presence of $\mathrm{H}_{2} \mathrm{O}_{2}$, and furthermore, the loss of

523 biofilm biomass by the $\Delta y f i N$ mutant in the absence of $\mathrm{H}_{2} \mathrm{O}_{2}$ could be rescued by replenishing

524 biofilms with fresh medium. These data suggest that YfiN may be important for adapting to

525 oxidative stressors generally present in the environmental milieu, including self-produced redox

526 active compounds produced by $P$. aeruginosa. Phenazines, small redox active molecules that can

527 act as alternate electron acceptors, may be one such molecule. Interestingly, phenazines have

528 been linked to EPS production via the DGC RmcA $(56,57)$ and we have shown that RmcA is

529 critical for biofilm maintenance in response to starvation (30), thus there are multiple lines of

530 evidence linking phenazines to the control of biofilm biology. Further work is needed to

531 understand if the ability for YfiN to regulate biofilm maintenance is specific to peroxide 
exposure or if $\mathrm{YfiN}$ is more broadly required across a range of redox active agents, including phenazines and immune system-generated molecules with similar chemistry (58-62). actively maintains a mature biofilm even in the face of environmental stressors. That is, $P$. aeruginosa appears to utilize at least three different c-di-GMP metabolizing enzymes, RmcA,

537 MorA and YfiN, to actively maintain the biofilm in response to environmental perturbations, 538 including starvation or peroxide exposure. Based on work here and our previous study of RmcA and MorA, the loss of the ability or modulate c-di-GMP in a mature biofilm in the face of

540 environmental stressors has dire consequences for the cell, that is, death. If one also considers

541 that published work showing that $P$. aeruginosa has pathways for the generation of "maintenance 542 energy" in the context of mature biofilms (63-65), it appears that this microbe has a robust set of 543 mechanisms to sense and respond to environmental inputs when in a biofilm, and then regulate

544 its response(s) appropriately. Such biofilm maintenance pathways might be key to preventing 545 biofilm bacteria from "over-reacting" to transient stresses; thus mediating persistence through 546 the perturbation without transitioning to a planktonic state. That is, perhaps biofilms have a type 547 of "fight" or "flight" response regulated by environmental inputs and c-di-GMP levels. We 548 speculate that such "biofilm maintenance" pathways are likely present in other microbes, and 549 furthermore, at least a subset of c-di-GMP-related enzymes for which there is no assigned 550 function play a role in such pathways.

A number of microbes have been shown to have the $y f i$ operon, which encodes the tri552 partite regulatory system of $Y$ fiN, YfiR and $Y$ fiB. The current model suggests that YfiR is 553 localized to the periplasm, where it directly inhibits Y fiN. This inhibition is relieved in the 554 presence of oxidative stress, which leads to misfolding of YfiR. Additionally, YfiB potentially 
sequesters YfiR following cell envelope stress, thus relieving inhibition of YfiN (47). Therefore, it is likely that both oxidative and cell envelope stress can regulate YfiN activity; our biofilm and motility results support the previously reported effects of oxidative stress (66). Moreover, YfiN has been reported to localize to the mid-cell and arrest division in Salmonella enterica and Escherichia coli and regulate cell division in response to reductive stress (67). Interestingly, increased mid-cell localization was not observed in $P$. aeruginosa (67), indicating that this protein contributes to different functions in different organisms.

The YfiBNR system shows conservation among other Gram-negative microbes beyond E. coli, including those organisms known to make biofilms. Serratia and Yersinia, also in the family Yersiniaceae, utilize the homologous system HmsCDE (38). Like YfiBNR, the HmsCDE system is implicated in biofilm growth and response to redox stress. Deletion of the $y f i N$ homolog, hmsD, in Y. pestis leads to decreases for in vitro and in vivo biofilm formation (68). Null mutants of $h m s D$ exhibit decreased biofilm biomass in reducing environments (69); these previous findings and our work here are consistent with the importance of YfiN in biofilm maintenance, particularly in response to redox conditions. Furthermore, in liquid culture, HmsD and $\mathrm{HmsC}$ (YfiR homolog) expression is more than doubled during the late stationary phase, and compared to the control, the $\Delta h m s D$ mutant exhibits a severe growth defect when grown in media containing $\mathrm{H}_{2} \mathrm{O}_{2}$. We did not observe increased sensitivity of the $\Delta y f i N$ mutant to $\mathrm{H}_{2} \mathrm{O}_{2}$ in planktonic conditions, again emphasizing the differential functions of this system in different organisms. 


\section{MATERIALS AND METHODS}

Strains and media. UCBPP-PA14 (PA14) was used in all the experiments. P. aeruginosa was

581 routinely streaked onto lysogeny broth (LB) plates containing $1.5 \%$ agar prior to overnight

582 culturing in LB liquid cultures at $37^{\circ} \mathrm{C}$. As needed, LB was supplemented with $10 \mathrm{mg} / \mathrm{ml}$

583 gentamicin $(\mathrm{Gm})$ and 50mg/ml kanamycin (Kan). Biofilm medium used in static 96-well crystal

584 violet assays was composed of M63 medium supplemented with $1 \mathrm{mM} \mathrm{MgSO} 4$ and $0.4 \%$

585 (wt/vol) L-arginine monochloride. For medium replacement assays, plates were grown as

586 indicated, spent medium discarded and biofilms washed twice prior to the addition of fresh

587 medium. To limit the formation bubbles caused by the addition of $\mathrm{H}_{2} \mathrm{O}_{2}$ during microfluidic-

588 based biofilm assays, we used buffered KA biofilm medium, a modification K10T medium that

589 contains 50mM Tris- $\mathrm{HCl}(\mathrm{pH} 7.4), 0.61 \mathrm{mM} \mathrm{MgSO} 4$, and 0.4\% arginine (70).

590 Static biofilm assays and quantification. Overnight cultures were inoculated into 96-well U-

591 bottom polystyrene plates (Costar) containing M63-based biofilm medium and grown for the

592 specified time at $37^{\circ} \mathrm{C}$ in a hydrated container prior to washing, staining with crystal violet (CV)

593 and solubilization of the CV stain with 30\% glacial acetic acid (71). The presence of the biofilm

594 was quantified by measuring the extent of biofilm-associated CV measured in a

595 spectrophotometer to determine the optical density at $500 \mathrm{~nm}$ (OD550).

For biofilms imaged microscopically in static assays, overnight cultures were prepared as

597 described above and inoculated into 8-well glass-bottom dishes containing M63 biofilm medium 598 and grown at $37^{\circ} \mathrm{C}$ for the desired length of time prior to imaging with a Nikon Eclipse Ti

599 inverted microscope where a minimum of 6 fields of view were captured. To assess the viability 
600 of biofilms grown in glass bottom dishes, growth medium was discarded, biofilms washed twice

601 with fresh medium, and the biofilms immersed in fresh medium containing propidium iodide 10

602 min prior to imaging. To assess the concentration of c-di-GMP we utilized $\mathrm{P}_{\mathrm{c} d r A}$ - $g f p$ fusion

603 expressed from a multicopy pMQ72 plasmid (72) which was maintained in overnight cultures

604 supplemented with Gm prior to inoculation into antibiotic-free M63 biofilm medium.

605 Microfluidics. Biofilms were visualized under flow in microfluidics chambers kindly provided

606 by the Nadell laboratory at Dartmouth College. Chambers used polydimethylsiloxane (PDMS)

607 bonded to a cover glass (1.5 $36 \mathrm{~mm} 60 \mathrm{~mm}$; Thermo Fisher, Waltham MA) through soft

608 lithography techniques $(73,74)$. Overnight bacterial cultures were centrifuged, resuspended in

609 biofilm medium, adjusted to an $\mathrm{OD}_{600}$ of 1, pipetted into microfluidics chambers, and allowed to

610 attach for $1 \mathrm{~h}$. Tubing (catalog no. 30 Cole Palmer PTFE) to transport influent and effluent

611 medium was attached first to BD 5-ml syringes containing biofilm medium, then to the

612 microfluidics chambers, and then to syringe pumps (Pico Plus Elite; Harvard Apparatus)

613 operating at a flow rate of $0.75 \mu 1 / \mathrm{min}$.

614 Image acquisition and data analysis. All microscope images were acquired using Nikon

615 Elements AR driving a Nikon Eclipse Ti inverted microscope and a Hamamatsu ORCA-Flash

6164.0 camera. Samples were imaged with either a Plan Apochromat 100 DM Oil or Plan Fluor 40

617 DIC M N2 objective. Fast scan mode and 2X2 binning were used for imaging. All images were

618 collected in a temperature controlled environmental chamber set to $37^{\circ} \mathrm{C}$. Images were processed

619 with background subtraction and signal strength quantified by measuring the mean signal

620 intensity/pixel through the Integrated Density (IntDen) function. To quantify single-cell motility,

621 images were inverted, a threshold applied and the TrackMate program (75) used. 
622 Statistical analysis. Data were analyzed using GraphPad Prism 8. Unless otherwise noted, data

623 are representative of the results from at least three independent experiments. A Student T-test

624 with a Dunnett's or Sidak's multiple comparisons post-test, as indicated, were used to compare

625 results and to assess significance.

626 Potential homolog identification and phylogenetic tree construction. The nucleotide

627 sequence of YfiBNR in PAO1 (NC_002516.2:1212717-1215074) was used as a query in a

628 Nucleotide BLAST search (40). Bacterial genomes within the RefSeq Representative genomes

629 database were searched with discontiguous megablast. The expectant threshold was set to

6300.0001 ; all other default algorithm parameters were used. Sequences with $>75 \%$ query cover

631 were aligned using MAFFT (41). FastTree 2.1.11 was used to construct a phylogenetic tree

632 based on the aligned sequences, using the GTR+CAT model and Gamma20-based likelihoods

633 (42). 1000 re-samples were used to calculate bootstrap support for the tree, and a

634 nonhomologous human protein was used as an outgroup to root the tree. Interactive Tree of Life

635 version 4 was used to visualize the phylogenetic tree.

636

637 Acknowledgements. We thank Carey Nadell for providing microfluidic devices, Zdenek

638 Svindrych for providing imaging guidance, Olga Zhaxybayeva for providing bioinformatic

639 advice, and Thomas Wood for generously and speedily sending strains. We also thank Gillian

640 Kenyon for transformation selected strains with fluorescent marker plasmids. This work was

641 supported by the NIH (R37 AI83256 to GAO) and the James O. Freedman Presidential Scholar

642 Award to SAK. 


\section{Literature Cited.}

646 1. Khan NH, Ishii Y, Kimata-Kino N, Esaki H, Nishino T, Nishimura M, Kogure K. 2007.

647 Isolation of Pseudomonas aeruginosa from open ocean and comparison with freshwater,

648 clinical, and animal isolates. Microb Ecol 53:173-186.

649 2. Donlan RM. 2001. Biofilms and device-associated infections. Emerg Infect Dis 7:277-

$650 \quad 281$.

651 3. Bhagirath AY, Li Y, Somayajula D, Dadashi M, Badr S, Duan K. 2016. Cystic fibrosis

652 lung environment and Pseudomonas aeruginosa infection. BMC Pulm Med 16:1-22.

653 4. Van Acker H, Van Dijck P, Coenye T. 2014. Molecular mechanisms of antimicrobial

654 tolerance and resistance in bacterial and fungal biofilms. Trends Microbiol 22:326-333.

655 5. Stewart PS, Franklin MJ, Williamson KS, Folsom JP, Boegli L, James GA. 2015.

656 Contribution of stress responses to antibiotic tolerance in Pseudomonas aeruginosa

657 biofilms. Antimicrob Agents Chemother 59:3838-3847.

658 6. Armbruster CR, Lee CK, Parker-Gilham J, de Anda J, Xia A, Tseng BS, Hoffman LR, Jin

659 F, Harwood CS, Wong GCL, Parsek MR. 2019. Heterogeneity in surface sensing

660 produces a division of labor in Pseudomonas aeruginosa populations. Elife 8:1-29.

661 7. Utada AS, Bennett RR, Fong JCN, Gibiansky ML, Yildiz FH, Golestanian R, Wong GCL.

662 2014. Vibrio cholerae use pili and flagella synergistically to effect motility switching and

663 conditional surface attachment. Nat Commun 5:1-8.

664 8. Luo Y, Zhao K, Baker AE, Kuchma SL, Coggan KA, Wolfgang MC, Wong GCL, aeruginosa surface behaviors. MBio 6:1-11.

667 9. Colvin KM, Gordon VD, Murakami K, Borlee BR, Wozniak DJ, Wong GCL, Parsek MR. 
2011. The Pel polysaccharide can serve a structural and protective role in the biofilm matrix of Pseudomonas aeruginosa. PLoS Pathog 7: e1001264.

10. Toutain CM, Caizza NC, Zegans ME, O’Toole GA. 2007. Roles for flagellar stators in biofilm formation by Pseudomonas aeruginosa. Res Microbiol 158:471-477.

11. Conrad JC, Gibiansky ML, Jin F, Gordon VD, Motto DA, Mathewson MA, Stopka WG, Zelasko DC, Shrout JD, Wong GCL. 2011. Flagella and pili-mediated near-surface singlecell motility mechanisms in P. aeruginosa. Biophys J 100:1608-1616.

12. Kuchma SL, Ballok AE, Merritt JH, Hammond JH, Lu W, Rabinowitz JD, O’Toole GA. 2010. Cyclic-di-GMP-mediated repression of swarming motility by Pseudomonas aeruginosa: The pilY1 gene and its impact on surface-associated behaviors. J Bacteriol 192:2950-2964.

13. Kulesekara H, Lee V, Brencic A, Liberati N, Urbach J, Miyata S, Lee DG, Neely AN, Hyodo M, Hayakawa Y, Ausubel FM, Lory S. 2006. Analysis of Pseudomonas aeruginosa diguanylate cyclases and phosphodiesterases reveals a role for bis-( $\left.3^{\prime}-5^{\prime}\right)$ cyclic-GMP in virulence. Proc Natl Acad Sci U S A 103:2839-2844.

14. Hickman JW, Tifrea DF, Harwood CS. 2005. A chemosensory system that regulates biofilm formation through modulation of cyclic diguanylate levels. Proc Natl Acad Sci U S A 102:14422-14427.

15. Baraquet C, Murakami K, Parsek MR, Harwood CS. 2012. The FleQ protein from Pseudomonas aeruginosa functions as both a repressor and an activator to control gene expression from the pel operon promoter in response to c-di-GMP. Nucleic Acids Res 40:7207-7218.

16. Friedman L, Kolter R. 2004. Genes involved in matrix formation in Pseudomonas 
aeruginosa PA14 biofilms. Mol Microbiol 51:675-690.

692 17. Paul R, Weiser S, Amiot NC, Chan C, Schirmer T, Giese B, Jenal U. 2004. Cell cycledependent dynamic localization of a bacterial response regulator with a novel di-guanylate cyclase output domain. Genes Dev 18:715-727.

18. Simm R, Morr M, Kader A, Nimtz M, Römling U. 2004. GGDEF and EAL domains inversely regulate cyclic di-GMP levels and transition from sessibility to motility. Mol

19. Hengge R. 2009. Principles of c-di-GMP signalling in bacteria. Nat Rev Microbiol 7:263273.

20. Danese PN, Pratt LA, Kolter R. 2000. Exopolysaccharide production is required for development of Escherichia coli K-12 biofilm architecture. J Bacteriol 182:3593-3596.

702 21. Ghafoor A, Hay ID, Rehm BHA. 2011. Role of exopolysaccharides in Pseudomonas aeruginosa biofilm formation and architecture. Appl Environ Microbiol 77:5238-5246.

704 22. Matz C, McDougald D, Moreno AM, Yung PY, Yildiz FH, Kjelleberg S. 2005. Biofilm

707 23. Flemming HC, Wingender J. 2001. Relevance of microbial extracellular polymeric substances (EPSs) - Part I: Structural and ecological aspects. Water Sci Technol 43:1-8.

709 24. Yan J, Nadell CD, Stone HA, Wingreen NS, Bassler BL. 2017. Extracellular-matrixmediated osmotic pressure drives Vibrio cholerae biofilm expansion and cheater exclusion. Nat Commun 8:1-11.

712 25. Jang IA, Kim J, Park W. 2016. Endogenous hydrogen peroxide increases biofilm 
Sci Rep 6:1-12.

715

716

717

718

720

721

722

723

724

725

726

727

728

729

730
26. Zhao Y, Cai Y, Chen Z, Li H, Xu Z, Li W, Jia J, Sun Y. 2021. SpoT-Mediated NapA upregulation promotes oxidative stress-induced Helicobacter pylori biofilm formation and confers multidrug resistance. Antimicrob Agents Chemother 65:e00152-21.

27. Wei Q, Le Minh PN, Dötsch A, Hildebrand F, Panmanee W, Elfarash A, Schulz S, Plaisance S, Charlier D, Hassett D, Häussler S, Cornelis P. 2012. Global regulation of gene expression by OxyR in an important human opportunistic pathogen. Nucleic Acids Res 40:4320-4333.

28. Hassett DJ, Cohen MS. 1989. Bacterial adaptation to oxidative stress: implications for pathogenesis and interaction with phagocytic cells. FASEB J 3:2574-2582.

29. Lau GW, Britigan BE, Hassett DJ. 2005. Pseudomonas aeruginosa OxyR is required for full virulence in rodent and insect models of infection and for resistance to human neutrophils. Infect Immun 73:2550-2553.

30. Katharios-Lanwermeyer S, Whitfield GB, Howell PL, O’toole GA. 2021. Pseudomonas aeruginosa uses c-di-GMP phosphodiesterases RmcA and MorA to regulate biofilm maintenance. MBio 12: e03384-20.

31. Ha DG, Richman ME, O’Toole GA. 2014. Deletion mutant library for investigation of functional outputs of cyclic diguanylate metabolism in Pseudomonas aeruginosa PA14. Appl Environ Microbiol 80:3384-3393.

32. Ueda A, Wood TK. 2009. Connecting quorum sensing, c-di-GMP, Pel polysaccharide, and biofilm formation in Pseudomonas aeruginosa through tyrosine phosphatase TpbA (PA3885). PLoS Pathog 5:1-15.

33. Malone JG, Jaeger T, Spangler C, Ritz D, Spang A, Arrieumerlou C, Kaever V, 
Landmann R, Jenal U. 2010. YfiBNR mediates cyclic di-GMP dependent small colony variant formation and persistence in Pseudomonas aeruginosa. PLoS Pathog 6:1-17.

34. Malone JG, Jaeger T, Manfredi P, Dötsch A, Blanka A, Bos R, Cornelis GR, Häussler S, Jenal U. 2012. The YfiBNR signal transduction mechanism reveals novel targets for the evolution of persistent Pseudomonas aeruginosa in cystic fibrosis airways. PLoS Pathog

35. Yang X, Yang XA, Xu M, Zhou L, Fan Z, Jiang T. 2015. Crystal structures of YfiR from Pseudomonas aeruginosa in two redox states. Biochem Biophys Res Commun 461:14-20.

36. Giddens SR, Jackson RW, Moon CD, Jacobs MA, Zhang X-X, Gehrig SM, Rainey PB. 2007. Mutational activation of niche-specific genes provides insight into regulatory networks and bacterial function in a complex environment. Proc Natl Acad Sci 104:18247-18252.

37. Sanchez-Torres V, Hu H, Wood TK. 2010. GGDEF proteins YeaI, YedQ, and YfiN reduce early biofilm formation and swimming motility in Escherichia coli. Appl Microbiol Biotechnol 2010902 90:651-658.

38. Ren GX, Yan HQ, Zhu H, Guo XP, Sun YC. 2014. HmsC, a periplasmic protein, controls biofilm formation via repression of $\mathrm{HmsD}$, a diguanylate cyclase in Yersinia pestis. Environ Microbiol 16:1202-1216.

755 39. Hufnagel DA, DePas WH, Chapmana MR. 2014. The disulfide bonding system suppresses CsgD-Independent cellulose production in Escherichia coli. J Bacteriol

40. Altschul SF, Madden TL, Schäffer AA, Zhang J, Zhang Z, Miller W, Lipman DJ. 1997. 
Nucleic Acids Res 25: 3389-3402.

761 41. Katoh K, Rozewicki J, Yamada KD. 2018. MAFFT online service: Multiple sequence alignment, interactive sequence choice and visualization. Brief Bioinform 20:1160-1166.

42. Price MN, Dehal PS, Arkin AP. 2010. FastTree 2 - Approximately maximum-likelihood trees for large alignments. PLoS One 5:e9490.

43. Maki DG, Hennekens CG, Phillips CW, Shaw W V., Bennett J V. 1973. Nosocomial urinary tract infection with Serratia marcescens: An epidemiologic study. J Infect Dis 128:579-587.

768

769

770

771

772

773

44. yu VL. 1979. Serratia marcescens: Historical perspective and clinical review. N Engl J Med 300:887-893.

45. Kalivoda EJ, Stella NA, O’Dee DM, Nau GJ, Shanks RMQ. 2008. The cyclic AMPdependent catabolite repression system of Serratia marcescens mediates biofilm formation through regulation of type 1 fimbriae. Appl Environ Microbiol 74:3461-3470.

46. Letunic I, Bork P. 2019. Interactive Tree Of Life (iTOL) v4: recent updates and new developments. Nucleic Acids Res 47:W256-W259.

47. Giardina G, Paiardini A, Fernicola S, Franceschini S, Rinaldo S, Stelitano V, Cutruzzolà F. 2013. Investigating the allosteric regulation of YfiN from Pseudomonas aeruginosa: Clues from the structure of the catalytic domain. PLoS One 8:1-15.

48. Le Guyon S, Simm R, Rehn M, Römling U. 2015. Dissecting the cyclic diguanylate monophosphate signalling network regulating motility in Salmonella enterica serovar Typhimurium. Environ Microbiol 17:1310-1320.

49. Wolfe AJ, Visick KL. 2008. Get the message out: Cyclic-di-GMP regulates multiple levels of flagellum-based motility. J Bacteriol 190:463-475. 
783 50. Kuchma SL, Brothers KM, Merritt JH, Liberati NT, Ausubel FM, O’Toole GA. 2007. BifA, a cyclic-di-GMP phosphodiesterase, inversely regulates biofilm formation and swarming motility by Pseudomonas aeruginosa PA14. J Bacteriol 189:8165-8178.

51. Krasteva P V., Fong JCN, Shikuma NJ, Beyhan S, Navarro MVAS, Yildiz FH, directly sensing cyclic di-GMP. Science 327:866-868.

52. Fernandez NL, Waters CM. 2019. Cyclic di-GMP increases catalase production and hydrogen peroxide tolerance in Vibrio cholerae. Appl Environ Microbiol 85:e01043-19.

53. Strempel N, Nusser M, Neidig A, Brenner-Weiss G, Overhage J. 2017. The oxidative

Pseudomonas aeruginosa. Front Microbiol 0:2311.

54. Poudyal B, Sauer K. 2018. The PA3177 gene encodes an active diguanylate cyclase that contributes to biofilm antimicrobial tolerance but not biofilm formation by Pseudomonas

55. Chua SL, Ding Y, Liu Y, Cai Z, Zhou J, Swarup S, Drautz-Moses DI, Schuster SC,

800 56. Dietrich LEP, Okegbe C, Price-Whelan A, Sakhtah H, Hunter RC, Newmana DK. 2013.

803 57. Okegbe C, Fields BL, Cole SJ, Beierschmitt C, Morgan CJ, Price-Whelan A, Stewart RC, 
114:E5236-E5245.

807 58. Panmanee W, Hassett DJ. 2009. Differential roles of OxyR-controlled antioxidant enzymes alkyl hydroperoxide reductase $(\mathrm{AhpCF})$ and catalase (KatB) in the protection of Pseudomonas aeruginosa against hydrogen peroxide in biofilm vs. planktonic culture.

811 59. Thomson E, Brennan S, Senthilmohan R, Gangell CL, Chapman AL, Sly PD, Kettle AJ. 2010. Identifying peroxidases and their oxidants in the early pathology of cystic fibrosis. Free Radic Biol Med 49:1354-1360.

60. Galli F, Battistoni A, Gambari R, Pompella A, Bragonzi A, Pilolli F, Iuliano L, Piroddi M, Dechecchi MC, Cabrini G. 2012. Oxidative stress and antioxidant therapy in cystic fibrosis. Biochim Biophys Acta - Mol Basis Dis 1822:690-713.

817 61. Robinson JM. 2008. Reactive oxygen species in phagocytic leukocytes. Histochem Cell Biol 20081302 130:281-297.

819 62. Fang FC. 2004. Antimicrobial reactive oxygen and nitrogen species: concepts and controversies. Nat Rev Microbiol 2004210 2:820-832.

821 63. Goodwine J, Gil J, Doiron A, Valdes J, Solis M, Higa A, Davis S, Sauer K. 2019. Pyruvate-depleting conditions induce biofilm dispersion and enhance the efficacy of antibiotics in killing biofilms in vitr o and in vivo. Sci Rep 9:1-16.

824 64. Lin YC, Cornell WC, Jo J, Price-Whelan A, Dietrich LEP. 2018. The Pseudomonas aeruginosa complement of lactate dehydrogenases enables use of d- and l-lactate and metabolic cross-feeding. MBio 9:1-12.

827 65. Basta DW, Bergkessel M, Newman DK. 2017. Identification of fitness determinants 
66. Varghese A, Ray S, Verma T, Nandi D. 2020. Multicellular string-like structure formation by Salmonella typhimurium depends on cellulose production: Roles of diguanylate cyclases, YedQ and YfiN. Front Microbiol 11:1-15.

67. Kim HK, Harshey RM. 2016. A diguanylate cyclase acts as a cell division inhibitor in a two-step response to reductive and envelope stresses. MBio 7:e00822-16.

68. Sun YC, Koumoutsi A, Jarrett C, Lawrence K, Gherardini FC, Darby C, Hinnebusch BJ. 2011. Differential control of Yersinia pestis biofilm formation in vitro and in the flea vector by two c-di-gmp diguanylate cyclases. PLoS One 6: e19267.

69. Ren GX, Fan S, Guo XP, Chen S, Sun YC. 2016. Differential regulation of c-di-GMP metabolic enzymes by environmental signals modulates biofilm formation in Yersinia pestis. Front Microbiol 7:1-12.

70. Collins AJ, Pastora AB, Jarrod Smith T, O’Toole GA. 2020. MapA, a second large RTX adhesin conserved across the Pseudomonads, contributes to biofilm formation by Pseudomonas fluorescens. J Bacteriol: e00277-20.

71. O’Toole GA, Kolter R. 1998. Flagellar and twitching motility are necessary for Pseudomonas aeruginosa biofilm development. Mol Microbiol 30:295-304.

72. Shanks RMQ, Caiazza NC, Hinsa SM, Toutain CM, O’Toole GA. 2006. Saccharomyces cerevisiae-based molecular tool kit for manipulation of genes from Gram-negative bacteria. Appl Environ Microbiol 72:5027-5036.

73. Sia SK, Whitesides GM. 2003. Microfluidic devices fabricated in poly(dimethylsiloxane) for biological studies. Electrophoresis 24:3563-3576.

74. Weibel DB, DiLuzio WR, Whitesides GM. 2007. Microfabrication meets microbiology. Nat Rev Microbiol 5:209-218. 
bioRxiv preprint doi: https://doi.org/10.1101/2021.07.29.454409; this version posted July 30, 2021. The copyright holder for this preprint (which

was not certified by peer review) is the author/funder, who has granted bioRxiv a license to display the preprint in perpetuity. It is made available under aCC-BY-NC 4.0 International license.

852 75. Tinevez JY, Perry N, Schindelin J, Hoopes GM, Reynolds GD, Laplantine E, Bednarek

853 SY, Shorte SL, Eliceiri KW. 2017. TrackMate: An open and extensible platform for single-particle tracking. Methods 115:80-90.

855 